\title{
Estimação de Parâmetros Genéticos para Peso em Diferentes Idades para Animais da Raça Tabapuã ${ }^{1}$
}

\author{
Laila Talarico Dias $^{2}$, Lucia Galvão de Albuquerque ${ }^{3}$, Humberto Tonhati ${ }^{3}$, Rodrigo de Almeida Teixeira ${ }^{2}$
}

RESUMO - Foram estimados parâmetros genéticos para pesos do nascimento aos 570 dias de idade para 35.308 animais da raça Tabapuã, nascidos entre 1975 e 2000, pertencentes à ABCZ (Associação Brasileira de Criadores de Zebu) sob três modelos distintos. O modelo 1 incluiu o efeito genético aditivo direto como aleatório, além dos efeitos fixos de grupo de contemporâneos, definido pelas variáveis: proprietário, rebanho, criador, rebanho do criador, sexo, condição de criação, ano e mês de nascimento, ano e mês da pesagem, e os efeitos linear e quadrático de idade do bezerro e idade da vaca ao parto como covariáveis. O modelo 2 compreendeu, além dos efeitos supracitados, o efeito de ambiente permanente materno. O modelo 3 constou dos efeitos genético aditivo direto e materno e de ambiente permanente materno (aleatórios) e os mesmos incluídos no modelo 1 (fixos). De acordo com o teste de razão de verossimilhança, o modelo 3 foi o mais adequado para ajustar os efeitos estudados. As estimativas de herdabilidade direta foram baixas a moderadas $(0,08$ a 0,26$)$, decrescendo do nascimento às idades subseqüentes, com picos aos 90 e aos 180 dias de idade. Aos 345 dias de idade, ocorreu novo aumento nas estimativas, com menor oscilação entre as estimativas subseqüentes até 570 dias de idade. As estimativas de herdabilidade materna foram baixas, sendo maiores no período da desmama.

Palavras-chave: componentes de variância, gado de corte, herdabilidade

\section{Estimation of Genetic Parameters for Weight in Different Ages in Tabapuã Cattle}

\begin{abstract}
Weight records of 35.308 Tabapuã animals born between 1975 and 2000, made available by the Brazilian Zebu Breeders Association (ABCZ), were used to estimate genetic parameters for growth from birth to 570 days of age. Univariate analyses were used to fit three different models including fixed effects of contemporary group (owner, herd, sex, breeding management, year-month of birth and year-month of weight) and age of calf and age of dam (linear and quadratic) as covariates. Model 1 included only the direct genetic additive random effect; Model 2 considered both direct genetic additive and maternal permanent environmental random effects and Model 3 included the direct genetic additive, maternal, maternal permanent environmental random effects. The likelihood ratio test indicated Model 3 best fitted the data. Heritability estimates for direct genetic effects ranged between 0.08 and 0.26 , decreasing after birth along the growth period, but high heritability values were observed at 90, 180 and 345 days of age, and slight changes thereafter. In general, maternal heritability estimates were low, with higher values at the weaning age.
\end{abstract}

Key Words: beef cattle, heritability, variance components

\section{Introdução}

As características de crescimento, além de serem influenciadas pelo genótipo do animal, também são afetadas por efeitos de ambiente. O crescimento prédesmama está fortemente relacionado ao potencial de crescimento pré e pós-natal do bezerro e à habilidade materna da vaca. Entre o nascimento e a desmama, a mãe pode influenciar o fenótipo de sua progênie pela qualidade e quantidade de proteção oferecida, pela modificação de seu comportamento e pela quantidade e/ou qualidade de nutrição fornecida pela produção de leite, sendo este o fator mais importante para a determinação do crescimento do animal (Hohenboken,
1985). A expressão da habilidade materna no desempenho do bezerro pode ser confundida com a expressão dos genes para crescimento recebidos de seus pais. A separação dos efeitos genéticos aditivos direto e materno e de ambiente permanente da mãe não é simples, nem mesmo em análises unicaracterísticas, uma vez que são necessárias diversas relações de parentesco, o que nem sempre é possível obter em dados de campo (Gerstmayr, 1992; Meyer, 1992; Robinson, 1996ab; Meyer \& Hill, 1997).

O conhecimento do comportamento dos componentes de variância ao longo da trajetória do crescimento de um animal, bem como a mudança na variação de acordo com a idade, é de grande importância

\footnotetext{
${ }^{1}$ Parte da tese de doutorado da primeira autora, financiada pela CAPES

2 Professor da Universidade de Brasília - Faculdade de Agronomia e Medicina Veterinária (FAV) - Caixa Postal 04508-DF, CEP: 70910-900. E.mail: laila@unb.br

${ }^{3}$ Professor Adjunto da Faculdade de Ciências Agrárias e Veterinárias (UNESP-Jaboticabal).
} 
para o melhoramento genético animal, pois, com essas informações, será possível identificar em que fase do crescimento existe maior variabilidade genética para a característica estudada e, conseqüentemente, a seleção dos animais com base nessas informações poderá ser mais eficiente.

Apesar de existirem vários trabalhos na literatura sobre estimativas de parâmetros genéticos para as raças zebuínas, até a década de 90, a maioria dos estudos era realizada com pequeno número de registros e os parâmetros genéticos eram, geralmente, estimados em idades pré-determinadas, além de não haver separação dos efeitos genéticos aditivos direto e materno. As avaliações dos componentes de (co)variância para características de crescimento, em geral, são obtidas considerando-se os pesos em idades padrão (peso ao nascimento, à desmama, ao sobreano e final) ou os ganhos de peso entre duas idades, mas as estimativas de parâmetros genéticos, incluindo efeito materno, para pesos em idades jovens em intervalo pequeno de idade são escassas na literatura (Albuquerque \& Meyer, 2001) e, para a raça Tabapuã, estas informações ainda não estão disponíveis.

Os objetivos neste trabalho foram definir modelos adequados que possibilitem descrever as estimativas de parâmetros genéticos, estimar os componentes de variâncias direta e materna e os coeficientes de herdabilidade para pesos do nascimento aos 570 dias de idade em animais da raça Tabapuã.

\section{Material e Métodos}

Foram utilizados dados de 54 rebanhos com registros de pesagens de 150.209 animais da raça Tabapuã, nascidos entre 1975 e 2000, pertencentes ao banco de dados oficial da Associação Brasileira dos Criadores de Zebu (ABCZ), mensurados ao nascimento e, em média, a cada 90 dias, até dois anos de idade.

A edição dos dados foi realizada no programa computacional SAS (SAS, 1998). Os arquivos foram montados em intervalos de 30 dias de idade, sobrepostos a cada 15 dias, criando-se as seguintes classes de idade: nascimento; 0 a 30 dias; 15 a 45 dias; 30 a 60 dias; 45 a 75 dias; e assim, sucessivamente, até 555 a 585 dias de idade, gerando 39 arquivos. O grupo de contemporâneos (GC) foi definido pelas variáveis proprietário, rebanho do proprietário, criador, rebanho do criador, sexo, condição de criação (mamando ou desmamado), ano e mês de nascimento, ano e mês da pesagem.
Durante a edição dos dados, foram retirados dos arquivos animais com pai e mãe desconhecidos, animais com peso ao nascer menor que $15 \mathrm{~kg}$ ou maior que $50 \mathrm{~kg}$, gêmeos, grupos de contemporâneos com menos de quatro animais, idade da vaca desconhecida ou menor que dois anos e intervalos de partos menores que nove meses. Para este trabalho, foram mantidos nos arquivos apenas os animais criados em pastagem.

No arquivo de pedigree, foram incluídas até três gerações de ancestrais e, após a edição do arquivo de dados, as análises unicaracterísticas foram realizadas com 35.308 animais, filhos de 806 touros e 12.398 vacas.

Foram considerados três modelos, diferenciados apenas pelos efeitos aleatórios. O primeiro modelo constou do efeito genético aditivo direto, como aleatório, além dos efeitos fixos de GC e dos efeitos linear e quadrático de idade do bezerro à pesagem e de idade da vaca ao parto, como covariáveis. O segundo modelo incluiu, além dos desses efeitos, o efeito de ambiente permanente materno. O terceiro modelo compreendeu os efeitos genéticos direto e materno e o efeito de ambiente permanente materno, além dos efeitos fixos constantes no primeiro modelo. Para a análise de peso ao nascer, a covariável idade do bezerro não foi incluída no modelo.

Os modelos podem ser representados na forma matricial como:

$$
\begin{aligned}
& \text { Modelo 1: } y=X b+Z a+e \\
& \text { Modelo 2: } y=X b+Z a+W c+e \\
& \text { Modelo 3: } y=X b+Z a+M m+W c+e
\end{aligned}
$$

em que: $y=$ vetor das variáveis dependentes; $b=$ vetor de efeitos fixos; $a=$ vetor de valores genéticos aditivos dos animais; $m=$ vetor de valores genéticos aditivos maternos; $c=$ vetor de efeitos de ambiente permanente da vaca; $e$ = vetor de efeitos residuais; $X$, $Z, M$ e $W$ são as matrizes de incidência respectivas para cada efeito.

Pressuposições:

$$
\begin{aligned}
& \mathrm{E}(y)=X \beta \\
& \mathrm{E}(a)=0, \mathrm{E}(m)=0, \mathrm{E}(c)=0 \text { e } \mathrm{E}(e)=0 . \\
& \operatorname{Var}\left[\begin{array}{l}
a \\
m \\
c \\
e
\end{array}\right]=\left[\begin{array}{cccc}
A \sigma_{a}^{2} & 0 & 0 & 0 \\
0 & A \sigma_{m}^{2} & 0 & 0 \\
0 & 0 & I_{n v} \sigma_{c}^{2} & 0 \\
0 & 0 & 0 & I_{n b} \sigma_{e}^{2}
\end{array}\right]
\end{aligned}
$$


em que: $A=$ matriz de parentesco genético aditivo; $I_{n v}$ e $I_{n b}=$ matrizes identidade de dimensão número de mães (nv) e total de observações (nb); $\sigma_{a}^{2}=$ variância genética aditiva direta; $\sigma_{\mathrm{m}}{ }^{2}=$ variância genética aditiva materna; $\sigma_{\mathrm{c}}^{2}=$ variância de ambiente permanente materno; $\sigma_{\mathrm{e}}^{2}=$ variância residual.

Os componentes de variância foram estimados pelo método da máxima verossimilhança restrita (Patterson \& Thompson, 1971), que utiliza algoritmo livre de derivadas, disponível no programa computacional MTDFREML (Boldman et al., 1993).

O teste de razão de verossimilhança (Likelihood ratio test - LRT) foi utilizado para determinação do modelo mais adequado para o conjunto de dados estudado, comparando-se o aumento na função de máxima verossimilhança (-2 log L) causada pela adição de um parâmetro ao modelo a uma distribuição de Qui-quadrado com “g” graus de liberdade e probabilidade de erro de 5\% (Hogg \& Craig, 1995).

\section{Resultados e Discussão}

As médias de peso variaram de $31,47 \mathrm{~kg}$ ao nascimento, sendo que todos os animais tinham esta informação, até 311,38 kg aos 570 dias de idade. Observou-se aumento praticamente linear do peso de acordo com a idade do animal até a desmama (em torno de 240 dias) e, após este período, pequena redução na taxa de ganho de peso (Figura 1 ).

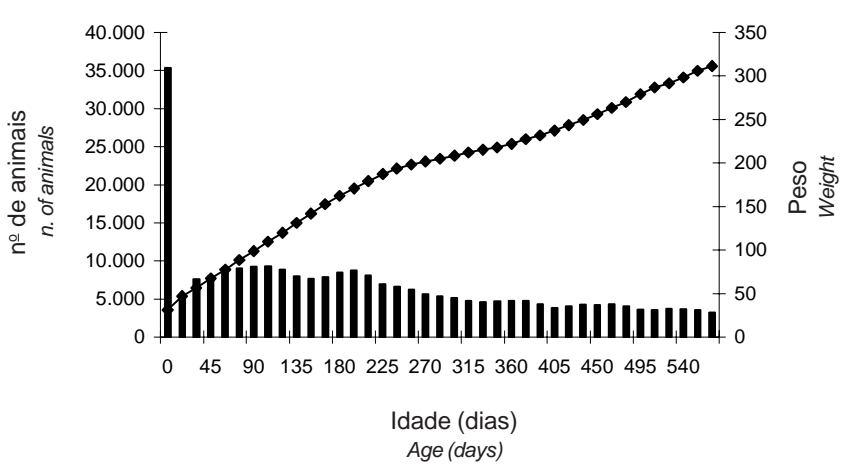

Figura 1 - Número de animais (-) e média de peso, em $\mathrm{kg}(\diamond)$ para cada idade, em dias.

Figure 1 - Number of animals (•) and mean weight, in $\mathrm{kg}(\bullet)$ for age, in days.
Na Figura 2 são apresentados os desvios-padrão e os coeficientes de variação para cada idade. Verificou-se aumento do coeficiente de variação do nascimento $(11,28 \%)$ até o primeiro mês de idade (21,67\%). A partir daí, houve decréscimo até por volta dos 105 dias (18,00\%), permanecendo praticamente constante após esse período. Houve também tendência de crescimento do desvio-padrão, variando de 3,55 kg ao nascimento a 57,25 kg aos 570 dias de idade. O menor valor do coeficiente de variação para o peso ao nascer pode ser explicado pela possível utilização da média de peso para esta idade.

O teste de razão de verossimilhança comprovou diferença significativa $(\mathrm{P}<0,05)$ entre os três modelos estudados até cerca de um ano de idade (345 dias), evidenciando a importância de se incluir os efeitos maternos (genético materno e ambiente permanente materno) nas análises para características de crescimento. Entre 345 e 420 dias de idade, os modelos 2 e 3 não diferiram estatisticamente entre si, o que pode ter ocorrido em razão do pequeno número de bezerros por vaca para estas idades (1,10 bezerros/vaca). Após os 420 dias de idade, os modelos 1, 2 e 3 não diferiram estatisticamente entre si. Resultados semelhantes foram reportados por Meyer (1992), que, analisando registros de crescimento de animais Bos taurus taurus e Bos taurus indicus sob seis modelos distintos, observou que o modelo que incluiu efeitos genéticos direto e materno e o efeito de ambiente permanente materno proporcionou melhor ajuste dos

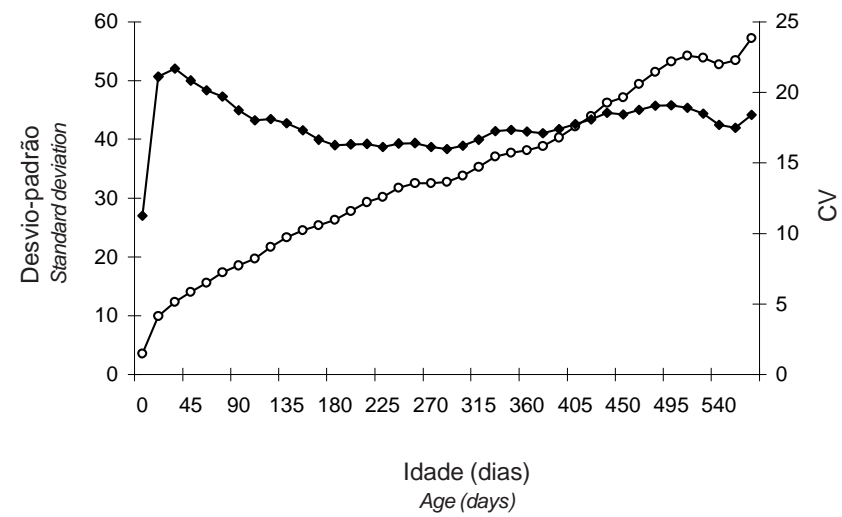

Figura 2 - Desvio-padrão, em kg (o) e coeficiente de variação, em \% ( $\bullet$ ) para cada idade, em dias.

Figure 2 - Standard deviation, in $\mathrm{kg}$ (0) and coefficient of variation $-C V$, in $\%(\bullet)$ for each age, in days. 
dados do nascimento até idade de abate, para animais da raça Hereford e cruzados com Zebu. Porém, para animais da raça Angus, após a desmama, os efeitos maternos tiveram menor importância.

Na Figura 3 são apresentadas as estimativas das variâncias fenotípicas e residuais para os modelos 1, 2 e 3 .

Para os três modelos estudados, os menores valores de variância fenotípica obtidos ocorreram no início do crescimento (em torno de $8,4 \mathrm{~kg}^{2}$ ) com crescimento contínuo de acordo com a idade até $1.050,0 \mathrm{~kg}^{2}$ no final do período estudado, aos 570 dias de idade. Independentemente do modelo empregado, o comportamento e a magnitude das variâncias fenotípicas foram praticamente os mesmos (Figura 3). Entretanto, Cyrillo (2003), ao estudar três modelos distintos para analisar o crescimento do nascimento até 13 meses de idade de bovinos machos da raça Nelore, observou que o modelo mais simples, que incluiu apenas o efeito genético aditivo direto, apresentou estimativas de variância fenotípica ligeiramente maiores que os modelos que incluíam os efeitos maternos.

As estimativas das variâncias residuais foram altas e semelhantes para os três modelos em todo o período estudado, chegando a $75 \%$ da variação total. Essa semelhança entre os resíduos dos três modelos estudados sugere que a inclusão dos efeitos maternos no modelo levou à partição do efeito genético. Resultado semelhante foi observado por Albuquerque \& Meyer (2001) para dados de crescimento de animais da raça Nelore.

Na Figura 4 encontram-se as variâncias genética aditiva direta, materna e de ambiente permanente para os modelos 1, 2 e 3 .

As estimativas dos componentes de variância do efeito direto obtidas com os modelos que incluíram os efeitos maternos (Modelos 2 e 3) foram bastante semelhantes. A não-inclusão dos efeitos maternos nos modelos de análise pode fazer com que as variâncias de ambiente permanente materno e genético materno sejam absorvidas na variância de efeito genético direto (Modelo 1 - Figura 4), ressaltando-se a importância da inclusão dos efeitos maternos nas análises para características de crescimento. No modelo 3 (modelo completo), pode-se observar menor estimativa de variância genética aditiva ao nascimento $2,23 \mathrm{~kg}^{2}$, em comparação aos demais modelos estudados (3,74 kg² para os modelos 1 e 2) e, após esta idade, valores crescentes para as idades subseqüentes. As estimativas de variância para ambiente permanente materno $\left(\sigma_{\mathrm{C}}{ }^{2}\right)$ aumentaram até os 225 dias de idade,
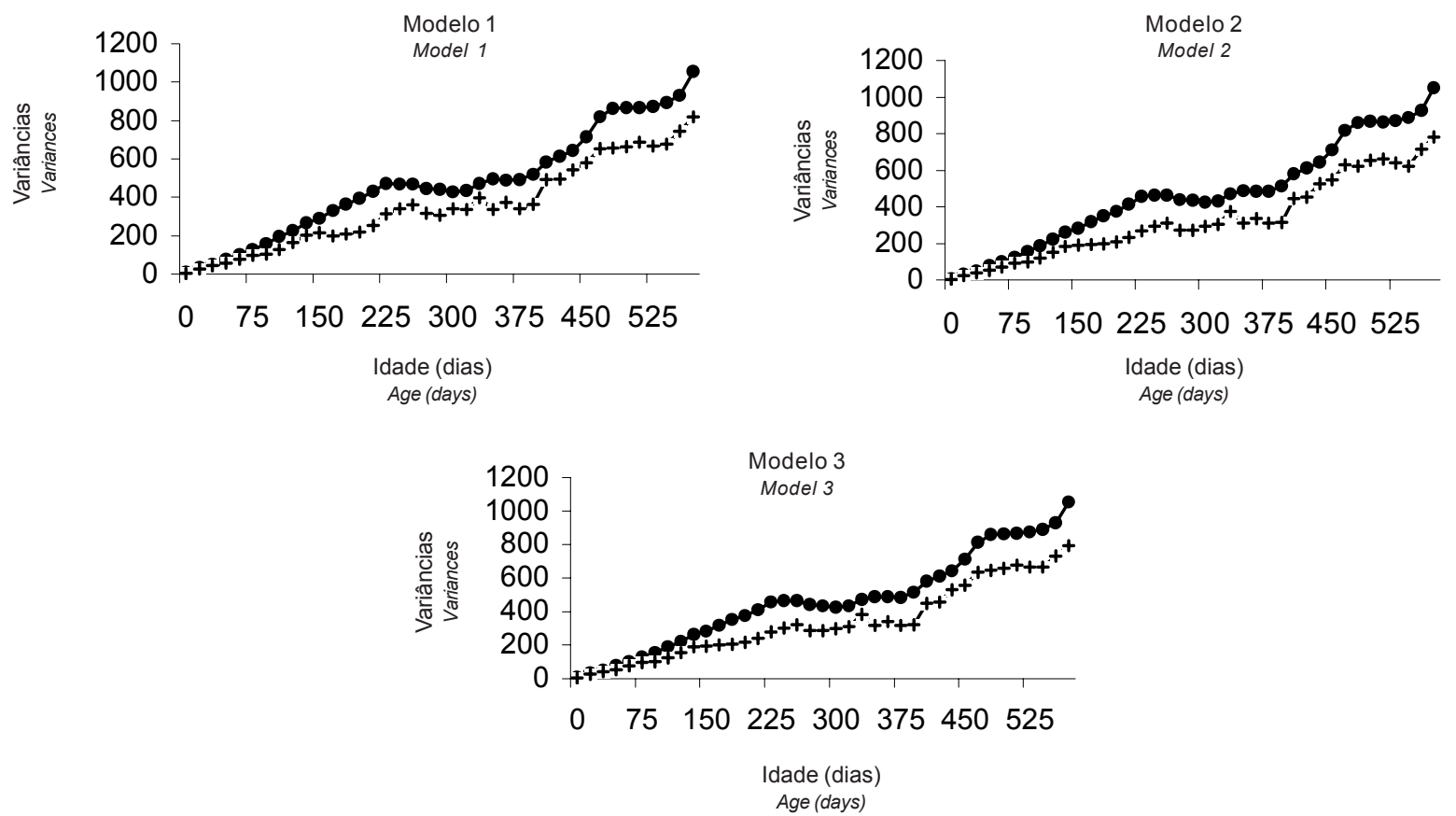

Figura 3 - Estimativas de variâncias fenotípicas (• ) e residuais (+), em $\mathrm{kg}^{2}$, para os modelos 1, 2 e 3, respectivamente. Figure 3 - Estimates of phenotypic (•) and residual variances (+), in $\mathrm{kg}^{2}$, for models 1,2 and 3, respectively. 

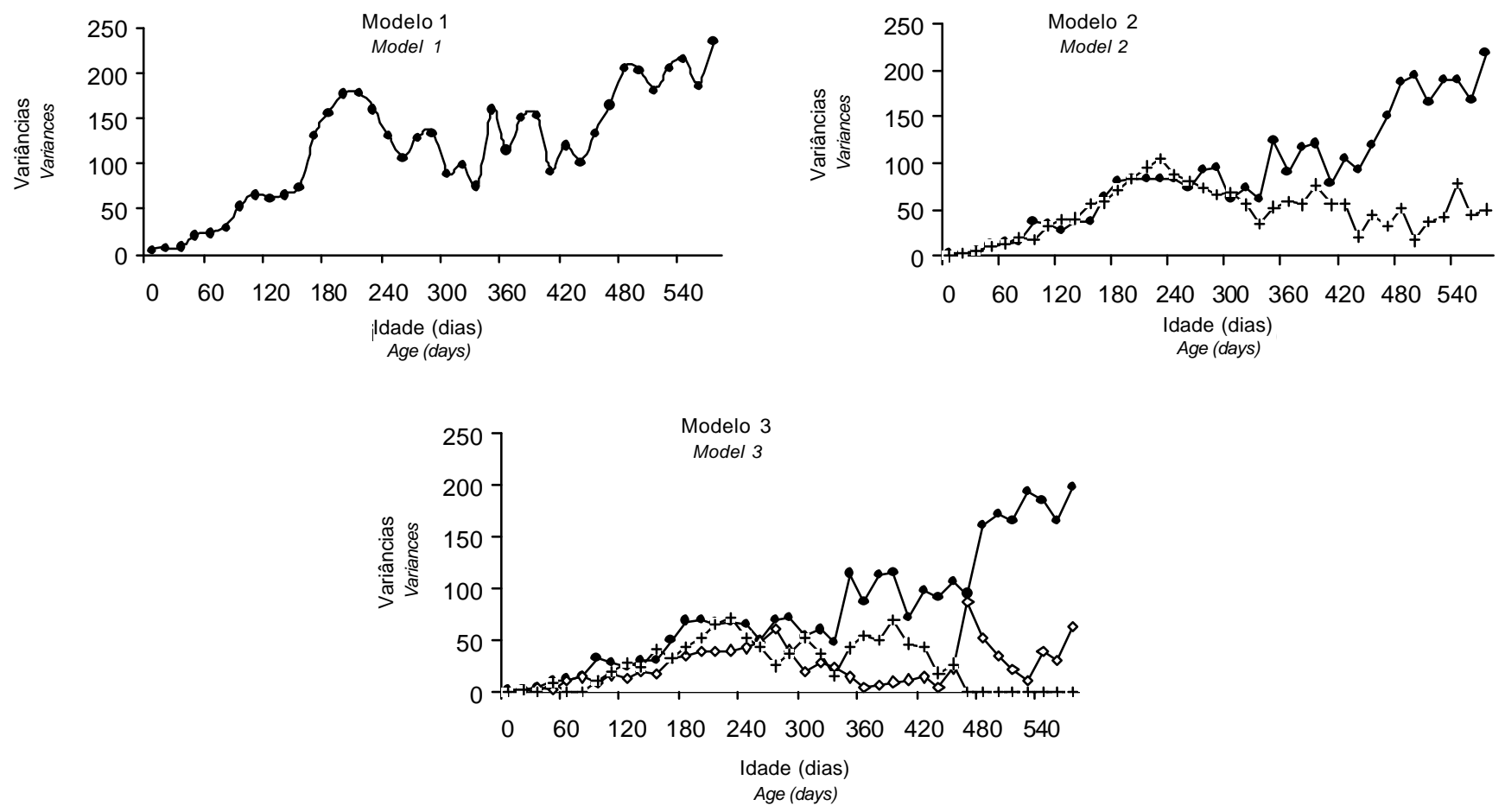

Figura 4 - Estimativas de variância genética aditiva direta $\sigma_{\mathrm{a}}{ }^{2}(\bullet)$ e materna $\sigma_{\mathrm{m}}{ }^{2}(\diamond)$ e de ambiente permanente materno $\sigma_{\mathrm{c}}{ }^{2}(+)$, em $\mathrm{kg}^{2}$, para os três modelos estudados.

Figure 4 - Estimates of genetic additive direct $\sigma_{a}^{2}(\bullet)$, maternal $\sigma_{m}^{2}(\diamond)$ and maternal permanent environmental $\sigma_{c}^{2}(+)$ variances, in $\mathrm{kg}^{2}$, for the three models.

diminuindo em seguida. A partir dos 330 dias de idade, as estimativas voltaram a aumentar até chegar aos 390 dias de idade, diminuindo gradativamente após esse período. De acordo com Meyer (1993), a influência dos efeitos maternos sobre os pesos após a desmama é conseqüência de efeitos em pesos anteriores a estas idades. Albuquerque \& Meyer (2001) observaram, em animais da raça Nelore, que as estimativas de variância de ambiente permanente materno aumentaram até os 150 dias de idade, mantendo-se praticamente constantes até os 240 dias, diminuindo após esta idade. Entretanto, Cyrillo (2003) relatou altas estimativas de variâncias de ambiente permanente materno, com crescimento contínuo até os 6-7 meses de idade, superiores às estimativas de variância genética aditiva direta, diminuindo gradualmente após este período, para machos da raça Nelore.

As estimativas de herdabilidades direta $\left(\mathrm{h}^{2}\right)$ e materna $\left(\mathrm{h}_{\mathrm{m}}{ }^{2}\right.$ ) para o modelo completo (modelo 3) são apresentadas na Figura 5.

As estimativas de herdabilidade direta foram baixas a moderadas e oscilaram muito em torno da linha de tendência. Foram encontrados maiores valores de herdabilidade no início e no final do período estudado, sendo a maior estimativa para o peso ao nascer $(0,26)$. Após o nascimento, as estimativas decresceram até próximo à desmama $(0,11)$, por volta de 240 dias, voltando a aumentar com a idade, chegando a 0,21 aos 570 dias. Os erros-padrão para as estimativas de herdabilidade obtidas neste trabalho variaram de 0,023 a 0,053 . Resultados semelhantes foram reportados por Albuquerque \& Meyer (2001), que observaram a maior estimativa de herdabilidade para o peso ao nascer $(0,28)$. Após o nascimento, os autores relataram a diminuição das estimativas até próximo ao período da desmama $(0,13)$, mas as estimativas aumentaram com a idade, chegando a 0,25 aos 570-600 dias de idade, para animais da raça Nelore. As estimativas obtidas neste trabalho comprovam tendência similar à relatada em revisão realizada por Mercadante et al. (1995), com valores maiores para peso ao nascer e aos 365 dias de idade e menores à desmama (0,29; 0,22 e 0,12, respectivamente). Lôbo et al. (2000) relataram valores médios de herdabilidade para peso ao nascer, à desmama e aos 365 dias de idade de 0,33; 0,22 e 0,20, respectivamente, para populações zebuínas, próximos dos resultados encontrados neste estudo. 
As estimativas de herdabilidade materna aumentaram do nascimento $(0,08)$ até próximo à desmama $(0,14)$, diminuindo nas idades subseqüentes, chegando a 0,06 aos 570 dias de idade. Esses resultados confirmam a maior importância do efeito materno até a desmama. Em idades subseqüentes, embora sua relevância diminua, este efeito continua presente, conforme demonstrado por Albuquerque \& Meyer (2001), ao estimarem os efeitos genéticos diretos e maternos para animais da raça Nelore.

\section{Conclusões}

O modelo que considerou os efeitos aleatórios genético aditivo direto, genético materno e de ambiente permanente materno foi o mais adequado para ajustar os efeitos estudados. Portanto, os efeitos maternos devem ser considerados na avaliação das características de crescimento de animais da raça Tabapuã, mesmo após o período da desmama.

As estimativas de herdabilidade direta foram baixas a moderadas, sendo maiores ao nascimento e no final do período estudado. As estimativas de herdabilidade materna foram baixas, sendo maiores no período da desmama.

\section{Literatura Citada}

ALBUQUERQUE, L.G.; MEYER, K. Estimates of direct and maternal genetic effects for weights from birth to 600 days of age in Nelore cattle. Journal of Animal Breeding and Genetics, v.118, p.83-92, 2001

BOLDMAN, K.G.; KRIESE, L.A.; Van VLECK, L.D. et al. A manual for use of MTDFREML. Clay Center: USDA-ARS, 1993. 120p.

CYRILLO, J.N.S.G.; ALENCAR, M.M.; RAZOOK, A.G. et al. Modelagem e estimação de parâmetros genéticos e fenotípicos para pesos do nascimento à seleção (348 dias) de machos Nelore. Revista Brasileira de Zootecnia, v.33, n.6, p.1405-1415, 2004
GERSTMAYR, S. Impact of the data structure on the reliability of the estimated genetic parameters in an animal model with maternal effects. Journal of Animal Breeding and Genetics, v.109, p.321-336, 1992.

HOGG, R.V.; CRAIG, A.T. Introduction to mathematical statistics. 5.ed. New Jersey: Prentice Hall, 1995. 564p.

HOHENBOKEN, W.D. Genetic structure of population. 7. Maternal effects. In: CHAPMAN, A.B. (Ed.) General and quantitative genetics. Amsterdan: Elsevier, 1985. p.135-146.

LÔBO, R.N.B.; MADALENA, F.E.; VIEIRA, A.R. Average estimates of genetic parameters for beef and dairy cattle in tropical regions. Animal Breeding Abstracts, v.68, p.433462, 2000.

MERCADANTE, M.E.Z.; LÔBO, R.B.; REYES, A. de los. Parámetros genéticos para características de crecimiento en cebuínos de carne. Archivos Latinoamericano Producción Animal, v.3, p.45-89, 1995.

MEYER, K. Variance components due to direct and maternal effects for growth traits of Australian beef cattle. Livestock Production Science, v.31, p.179-204, 1992.

MEYER, K. Estimates of covariance components for growth traits of Australian Charolais cattle. Australian Journal of Agricultural Research, v.44, p.1501-1508, 1993.

MEYER, K.; HILL, W.G. Estimation of genetic and phenotypic covariance functions for longitudinal 'repeated' records by restrict maximum likelihood. Livestock Production Science, v.47, p.185-200, 1997.

PATTERSON, H.D.; THOMPSON, R. Recovery of inter-block information when block sizes are unequal. Biometrika, v.58, p.545-54, 1971.

ROBINSON, D.L. Estimation and interpretation of direct and maternal genetic parameters for weights of Australian Angus cattle. Livestock Production Science, v.45, p.1-12, 1996a.

ROBINSON, D.L. Models which might explain negative correlation between direct and maternal genetic effects Livestock Production Science, v.45, p.111-112, 1996b.

STATISTICAL ANALYSES SYSTEM - SAS. SAS/STAT. User's guide. version 6.12, 4.ed, v.2, Cary: 1998. 842p.

Recebido em: 14/09/04

Aceito em: 08/06/05 